\section{Previous acute polio and post-polio syndrome: recognizing the pathophysiolo- gy for the establishment of rehabilitation programs}

\author{
Marco Orsini, 1,2 Jano Alves de Souza,2 \\ Marco Antonio Araújo Leite, ${ }^{2}$ \\ Silmar Teixeira, 3 \\ Arthur de Sá Ferreira, 1 \\ Victor Hugo Bastos, 3 \\ Marcos RG de Freitas, 2 \\ Acary Bulle Oliveira ${ }^{4}$ \\ ${ }^{1}$ Rehabilitation Sciences, Augusto Motta \\ University Center, UNISUAN, \\ Bonsucesso; 2Neurology Service, \\ Fluminense Federal University, Niterói; \\ 3Physical Therapy Department, Federal \\ University of Piauí; ${ }^{4}$ Neurology Service, \\ Paulista School of Medicine, Federal \\ University of São Paulo, Brazil
}

Previous acute poliomyelitis (PAP) can be defined as an endemic human disease caused by an enterovirus of worldwide distribution, which compromises the anterior horn cells of the spinal cord. Poliovirus has infected and victimized thousands of people all over the world. Only after the development of the inactivated virus vaccine by Jonas Salk, in 1955, and then with the attenuated virus vaccine, by Albert Bruce Sabin, in 1961, we saw a reduction in the number of poliomyelitis cases in the world. ${ }^{1}$

The patients present clinical status characterized by muscle atrophy and paresis, especially in the lower limbs, under asymmetrical and disproportional form. There is a second form, bulbar, which compromises the motor neurons of the medulla, resulting in impairments in speech, swallowing and breathing. The purpose of this letter to the Editor is to alert readers about the risks of therapeutic exercise for this group of patients. ${ }^{2}$

The pathophysiological framework related to the genesis of post-polio syndrome (PPS) is based on the theory of super training. Shortly after the episode of (PAP), the remaining motor neurons increase the number of sprouts (sproutings) for reinnervation of muscle fibers once denervated. Years after the acute episode, about three decades, there is a tendency to overload this system - that can be accelerated or attenuated depending on individual factors and especially the activities and/or therapeutic exercises carried out. In this case an intense metabolic demand in residual motor units occurs, triggering a process of secondary neuronal death. It is believed that $70 \%$ of patients victimized by PAP the will develop PPS. $^{3}$

The PPS is marked by a new framework for amyotrophy, unusual fatigue, joint problems and pain - all of progressive character. The prevalence of fatigue is remarkably high, and may contribute significantly to patients' disability and a poor quality of life. Other possible manifestations are: intolerance to cold and sleep apnea. We emphasize that, although there is a wide range of signs and symptoms, its totality is not expressed in all those affected.

Aerobic training conducted at submaximal limits and muscle strengthening activities of low intensity have shown positive effects on muscle strength and cardio-respiratory system in patients with PPS. Therapeutic activities carried out in aquatic environments have a positive impact on pain and motor function. In patients with severe fatigue, it is recommended caution in the implementation of rehabilitation programs. Recognition of respiratory impairment and early introduction of non-invasive ventilatory aids prevent or delay further respiratory decline and the need for invasive respiratory aid. They must receive prophylactic treatment with antibiotics, immunization against flu and Pneumococcus and avoid smoking The management of patients by an interdisciplinary team is crucial for the establishment of goals in the short, medium and long term. 4

Today, the treatment of post-polio syndrome is based on non-pharmacological intervention, including lifestyle modification, joint protection, and the use of orthoses, adaptive equipment, or mobility aids that can reduce fatigue and preserve function. Decrease of physical activity, rest periods during the day and an individually tailored training program. Weight gain is to be avoided if at all possible in these patients, because increased weight only leads to further difficulty in the performance of daily activities. ${ }^{4}$

Pain control may be difficult because it is usually generalized and not limited to a joint or limb. Simple physical procedures, such as heat, cold, massage and passive stretching may be very helpful; transcutaneous electrical nerve stimulation and acupuncture may also help in treatment. In 2009, a group of researchers of Neurology Service from the Federal University of São Paulo developed a bioceramic fabric, MIG3, a long infrared emitter. The researchers came to the conclusion that, over four weeks, it had significantly reduced complaints of pain and improved sleep quality. The radiation emitted by long infrared (LIR) fabrics increases membrane permeability and the calcium concentration in the cell membrane and cytoplasm; it biologically stimulates the cell, favoring the exchange of metabolites within the extracellular medium, nurturing the cell. It also acts on mitochondria, promoting the ATP synthesis used in muscular contraction. 5

There is no diagnostic test for PPS, and the diagnosis is based on a proper clinical workup where all other possible explanations for the new
Correspondence: Marco Orsini, Programa de PósGraduação em Ciências da Reabilitação, Praça das Nações, 34, Bonsucesso, Rio de Janeiro, CEP: 21041021, Brasil.

E-mail: orsinimarco@hotmail.com

Received for publication: 5 November 2014.

Accepted for publication: 3 January 2015

This work is licensed under a Creative Commons Attribution NonCommercial 3.0 License (CC BYNC 3.0).

(C) Copyright M. Orsini et al., 2015

Licensee PAGEPress, Italy

Neurology International 2015; 7:5452

doi:10.4081/ni.2015.5452

symptoms are ruled out. Intravenous immunoglobulin (IVIG) has been tried in some studies, all having positive results. IVIG could probably be a therapeutic alternative, but the potential benefit is modest and transitory. ${ }^{6}$

We end this editorial indicating that many patients ask us about the real effects of therapeutic exercise in the minimization or reversibility of the decline of their functions. We show that, in the general population, physical activity is considered as an adjunct to a good health condition and psychological benefits, provided that it respects the particularities of patients and especially the pathophysiological framework of PPS.

\section{References}

1. Lira CAB, Vancini RL, Cabral FR, et al. Post-polio syndrome: renaissance of poliomyelitis? Review einstein 2009;7:2258.

2. Atwal A, Giles A, Spiliotopoulou G, et al. Living with polio and postpolio syndrome in the United Kingdom. Scand J Caring Sci 2013;27:238-45.

3. Esteban J. [Polio paralytic. New problems: postpolio syndrome]. Rev Esp Salud Publica. 2013;87:517-22. [Article in Spanish].

4. Matyja E. Post-polio syndrome. Part II Therapeutic management. Neurol Neurochir Pol 2012;46:372-8.

5. Silva TM, Moreira GA, Quadros AA, et al Effects of the use of MIG3 bioceramics fabrics use - long infrared emitter - in pain, intolerance to cold and periodic limb movements in post-polio syndrome. Arq Neuropsiquiatr 2009;67:1049-53.

6. Bertolasi L, Frasson E, Turri M, et al. A randomized controlled trial of IV immunoglobulin in patients with postpolio syndrome. $\mathrm{J}$ Neurol Sci 2013;330:94-9. 